\title{
Drug-induced liver injury
}

\author{
Authors: Dev Katarey ${ }^{A}$ and Sumita Verma ${ }^{B}$
}

Drug-induced liver injury (DILI) remains the most common cause of acute liver failure (ALF) in the western world. Excluding paracetamol overdose, nearly all DILI encountered in the clinical setting is idiosyncratic in nature because affected individuals represent only a small proportion of those treated with such drugs. In many cases, the mechanism for idiosyncrasy is immune-mediation and is often identified by genetic risk determined by human leukocyte antigen variants. In the absence of diagnostic tests and/or biomarkers, the diagnosis of DILI requires a high index of suspicion after diligently excluding other causes of abnormal liver tests. Antibiotics are the class of drugs most frequently associated with idiosyncratic DILI, although recent studies indicate that herbal and dietary supplements are an increasingly recognised cause. It is imperative that upon development of DILI the culprit drug be discontinued, especially in the presence of elevated transaminases (aspartate aminotransferase/alanine aminotransferase ratio $\geq 5$ times the upper limit of normal) and/or jaundice. Risk factors for the development ALF include hepatocellular DILI and female gender, the treatment being supportive with some benefit of $\mathrm{N}$-acetylcysteine in the early stages. In view of the poor transplant-free survival in idiosyncratic DILI, early consideration for liver transplant is mandatory.

KEYWORDS: Autoimmune hepatitis, cholestasis, drug-induced acute liver failure, herbal and dietary supplements, hepatocellular DILI, hepatotoxicity

\section{Introduction}

At a regulatory level, drug-induced liver injury (DILI) is the commonest reason for withdrawing drugs from the market and/or issuing warnings and modification of use. ${ }^{1}$ DILI is classified as either predictable or unpredictable (idiosyncratic). ${ }^{2}$ The former tends to be dose-related, has a short latency (days), is due to direct toxicity of the drug or its metabolite, and is

\footnotetext{
Authors: ${ }^{A}$ academic clinical fellow (hepatology) and honorary research fellow, Department of Medicine, Brighton and Sussex Medical School, and Department of Gastroenterology and Hepatology, Brighton and University Hospital, Brighton, UK; ${ }^{\text {B }}$ reader in medicine and honorary consultant in hepatology, Department of Medicine, Brighton and Sussex Medical School, and Department of Gastroenterology and Hepatology, Brighton and University Hospital, Brighton, UK
}

reproducible in animal models (eg paracetamol overdose). ${ }^{3}$ The vast majority of DILI, however, is idiosyncratic or unpredictable - it is unexpected based on the pharmacological actions of the drug and in most cases routine animal toxicology fails to identify the risk of subsequent clinical toxicity. ${ }^{4}$ This review shall largely focus on idiosyncratic DILI.

\section{Epidemiology}

Idiosyncratic DILI is an uncommon adverse event when taking prescription or non-prescription medications. It is difficult to assess the true incidence of DILI because of different diagnostic criteria and under reporting. ${ }^{5}$ However, recent populationbased studies estimate the incidence to vary between 13.9-19.1 cases per 100,000 people per year. ${ }^{5,6}$

Data from prospective DILI registries suggest that antibiotics remain the most common cause of idiosyncratic DILI. The American DILI Network (DILIN) reported antibiotics to be implicated in $45.4 \%$ of cases. ${ }^{7}$ Other common drug classes reported by the American DILIN were herbal and dietary supplements (HDS; 16.1\% - a significant increase over the last 10 years), cardiovascular agents $(9.8 \%)$, central nervous system agents $(9.1 \%)$, anti-neoplastic agents (5.5\%) and analgesics $(3.7 \%) .^{7}$ Amoxicillin-clavulanate is the most common individual drug implicated in DILI as confirmed by both European and American studies (Table 1) ${ }^{6-10}$ In some Asian countries, however, HDS are implicated in more than $70 \%$ of all DILI. ${ }^{11}$

DILI includes the whole spectrum from asymptomatic elevation in liver tests to acute liver failure (ALF). In fact, DILI remains the most common cause of ALF in the UK ${ }^{12}$ and USA. ${ }^{13}$ Specifically, in the UK, paracetamol overdose causes approximately $57 \%$ of all ALF with non-paracetamol drugs accounting for a further $11 \% .{ }^{12}$ In idiosyncratic DILI, approximately $4 \%$ progress to ALF; risk factors are female gender $(70 \%)$ and hepatocellular DILI $(80 \%){ }^{8}$ The drugs most commonly implicated in ALF include antituberculosis therapies (ATT), HDS, sulpha-containing drugs, nitrofurantoin, phenytoin, sodium valproate, flutamide and amoxicillin-clavulanate. ${ }^{8,14}$

\section{Types of DILI}

Idiosyncratic DILI can be classified according to the pattern of liver tests observed - hepatocellular, cholestatic, or mixed. This classification was first established by the Council for International Organizations of Medical Sciences (CIOMS) $)^{15}$ and has recently been modified by the US Food and Drug 
Table 1. Common drugs implicated in idiosyncratic drug-induced liver injury according to studies from different countries

\begin{tabular}{llll} 
Iceland $^{6}$ & American DILIN & Spanish registry $^{\mathbf{8}}$ & UK $^{9}$ \\
Amoxicillin-clavulanate & Amoxicillin-clavulanate & Amoxicillin-clavulanate & Amoxicillin-clavulanate \\
Diclofenac & Isoniazid & Isoniazid & Diclofenac \\
Azathioprine & Nitrofurantoin & Combined anti-tuberculous therapy & Tricyclic antidepressants \\
Infliximab & Trimethoprim-sulfamethoxazole & Flutamide & Macrolides \\
Nitrofurantoin & Minocycline & Ibuprofen & Chlorpromazine \\
\hline
\end{tabular}

DILIN = drug-induced liver injury network

Administration (FDA) Drug Hepatotoxicity Steering Committee. ${ }^{16}$ Hepatocellular DILI is characterised by an alanine aminotransferase (ALT) $\geq 3$ times the upper limit of normal (ULN) and ALT/alkaline phosphatase (ALP) ratio $\geq 5$ times ULN; cholestatic DILI by an ALP $\geq 2$ times ULN and ALT/ALP ratio of $\leq 2$ times ULN; and mixed DILI with ALT $\geq 3$ times ULN, ALP $\geq 2$ times ULN and ALT/ALP ratio $<5$ but $>2$ times ULN. Individual drugs have dispositions toward a characteristic clinical signature; however, exceptions do occur. For example, amoxicillin-clavulanate usually results in a cholestatic injury but less frequently has also been associated with ALF. ${ }^{17,18}$

Another way to classify idiosyncratic DILI is as immunemediated (allergic) or non-immune mediated (nonallergic). ${ }^{19}$ If immune-mediated, the latency is shorter (1-6 weeks) compared with non-immune mediated reactions (1 month to 1 year). ${ }^{1,20}$ However, exceptions do occur with immune reactions appearing after a very long latency with drugs such as nitrofurantoin ${ }^{21}$ or even after drug cessation (eg sulfonamides, erythromycin and amoxicillinclavulanate).$^{20}$ Immune-mediated idiosyncratic reactions can be characterised by presence of fever, rash, eosinophilia, autoantibodies (such as antinuclear and anti-smooth muscle antibodies) and Stevens-Johnson syndrome. Non-immune mediated reactions lack the aforementioned characteristics and importantly, as described, tend to have a long latency period ( 1 month to 1 year). ${ }^{1,4,20}$

Other forms of DILI include steatohepatitis (amiodarone, tamoxifen, methotrexate), ${ }^{22}$ neoplasms (hepatic adenomas due to androgenic anabolic steroids (AAS)), ${ }^{23}$ and vascular (nodular regenerating hyperplasia due to azathioprine). ${ }^{24}$

\section{Risk factors}

Multiple factors have been found to be associated with an increased susceptibility to idiosyncratic DILI. The American DILIN found a higher prevalence of DILI in women (59\% female versus $41 \%$ male), ${ }^{7}$ although this was not corroborated by the Spanish DILI registry ( $49 \%$ female versus $51 \%$ male). ${ }^{8}$ Potential reasons for increased female susceptibility to DILI include differences in various aspects of drug pharmacokinetics or pharmacodynamics; hormonal effects or interactions with immunomodulating agents or signaling molecules; and differences in the adverse response of the immune system to some drugs, reactive drug metabolites or drug-protein adducts. ${ }^{25}$ It is consistently observed, however, that women tend to be younger and develop hepatocellular DILI whereas men tend to be older and develop cholestatic DILI., ${ }^{7,26}$
Older patients have an almost threefold increase in the incidence of DILI as shown in a study from Iceland. ${ }^{6}$ This was attributed to the increased prescription rate of drugs in older patients. ${ }^{6}$ In general, higher drug dose and longer duration of therapy have also been implicated in poorer outcomes. ${ }^{26-28}$ In the Spanish Registry, $77 \%$ of patients with idiosyncratic DILI received medications with daily doses of $\geq 50 \mathrm{mg} .{ }^{26}$ Ninety percent of patients with serious DILI needing a liver transplant and reported to the Swedish Adverse Drug Reactions Advisory Committee were consuming $\geq 50 \mathrm{mg} /$ day of the drug. ${ }^{27} \mathrm{~A}$ longer duration of drug therapy ( $135 \pm 31$ days versus $53 \pm 3$ days, $\mathrm{p}<0.0001)$ has been associated with a higher liver-related morbidity and mortality. ${ }^{29}$

It remains controversial as to whether pre-existing liver disease increases susceptibility to idiosyncratic DILI. In the American DILIN, 10\% of patients with DILI had pre-existing liver disease, mostly due to hepatitis $\mathrm{C}$ and non-alcoholic fatty liver disease (NAFLD). This group was characterised by more severe liver injury and higher overall mortality $(16 \%$ verus $5.2 \%, \mathrm{p}<0.001)$ although they had similar liver-related mortality ( $57 \%$ versus $46 \%, p=0.5) .^{7}$ The higher overall mortality in those with pre-existing liver disease may have been due to significantly higher prevalence of comorbidities, specifically diabetes mellitus ( $38 \%$ versus $23 \%, p=0.004$ ). ${ }^{7}$ Additionally, alcohol excess and/or risk factors for NAFLD increase susceptibility to methotrexate toxicity by three- to fourfold. ${ }^{30}$ Furthermore, some studies have shown that patients with chronic hepatitis B or C could have a threefold increased risk of abnormal liver tests after receiving ATT. ${ }^{31,32}$ Nonetheless, the interpretation of liver injury in patients with chronic hepatitis $\mathrm{B}$ and $\mathrm{C}$ receiving ATT remains complex. It could be due to ATT-induced DILI, viral reactivation and/or immune reconstitution. ${ }^{33}$

Genetic factors also increase susceptibility to DILI. For example, human leukocyte antigen (HLA) B ${ }^{\star} 5701$ confers an eightyfold increased risk to flucloxacillin induced DILI, whereas the absence of this genotype has an $88 \%$ negative predictive value for flucloxacillin-related DILI. ${ }^{34}$

In case 1 (Box 1), we present a patient who could be considered to have flucloxacillin hepatotoxicity. However, unusual features were the young age, short duration of drug use and near normal gamma-glutamyl transferase despite significant hyperbilirubinaemia. A more detailed history revealed the use of the dietary supplement Massdrol (which is an AAS) prior to ingestion of flucloxacillin. A subsequent liver biopsy revealed bland cholestasis characteristic of AAS hepatotoxicity (Fig 1). ${ }^{35}$ His HLA genotype was B12/B42. HLA 
Box 1. Three cases of drug-induced liver injury what is the diagnosis and management?

\section{Case 1}

A 33-year-old male was admitted with a 2-week history of jaundice. He had been prescribed a 5-day course of flucloxacillin 3 weeks previously for an ingrown toenail.

Investigations demonstrated bilirubin $120 \mu \mathrm{mol} / \mathrm{L}$ (peak $614 \mu \mathrm{mol} / \mathrm{L})$, AST $244 \mathrm{iu} / \mathrm{L}$, ALT $312 \mathrm{iu} / \mathrm{L}$, ALP $130 \mathrm{iu} / \mathrm{L}, \gamma \mathrm{GT} 72 \mathrm{iu} / \mathrm{L}$ and INR 1.0. Liver screen, including viral serology and imaging, were unremarkable.

\section{Case 2}

A 62-year-old female was referred for abnormal liver tests: ALT $125 \mathrm{iu} / \mathrm{L}, \mathrm{ALP} 245 \mathrm{iu} / \mathrm{L}$. On examination, she had stigmata of chronic liver disease, and further investigation revealed a positive anti-smooth muscle antibody and elevated immunoglobulins, specifically IgG. Imaging confirmed cirrhosis. She had commenced a statin 1 year previously.

\section{Case 3}

A 55-year-old male, co-infected with the hepatitis B (inactive carrier) and hepatitis $C$ virus, was 4 months into a 6 -month course of pegylated interferon alpha and ribavirin. He was non-cirrhotic and thus far had shown an excellent response to antivirals with a normal liver panel and negative hepatitis $B$ and C PCR. He was subsequently prescribed a 7-day course of clarithromycin for a chest infection and presented a week later with jaundice and confusion.

Investigations demonstrated bilirubin $350 \mu \mathrm{mol} / \mathrm{L}$, ALT $2000 \mathrm{iu} / \mathrm{L}$ and INR 3.0. Liver screen, including viral PCR and imaging, were unremarkable.

$\mathrm{ALP}=$ alkaline phosphatase; $\mathrm{ALT}=$ alanine aminotransferase; $\mathrm{AST}=$ aspartate aminotransferase; $I N R=$ international normalised ratio; $\mathrm{PCR}=$ polymerase chain reaction; $\gamma \mathrm{GT}=$ gamma-glutamyl transferase

$B \star 5701$, which confers susceptibility to flucloxacillin-related hepatotoxicity, ${ }^{34}$ was not identified.

\section{Pathogenesis}

The pathogenesis of idiosyncratic DILI remains unknown but is most likely due to a complex interplay between drug (eg dose, duration of therapy, hepatic metabolism, lipophilicity) and host factors (eg age, gender, genetic polymorphisms). The drug may form a reactive metabolite that triggers DILI or may form covalent adducts with tissue proteins, eliciting an immune response and subsequent DILI. ${ }^{36,37}$

\section{Natural history of DILI, and Hy's Law}

In the American DILIN, the presentation of DILI was with jaundice in $70 \%$ of cases. Most recovered after drug withdrawal; however, $17 \%$ progressed to chronic DILI (defined as abnormal liver tests for longer than 6 months) and $10 \%$ needed a liver transplant and/or died. ${ }^{7}$ Mortality increased to $44 \%$ in the presence of Stevens-Johnson syndrome. ${ }^{7}$ Compared with those with cholestatic DILI, those with hepatocellular DILI were more likely to require a liver transplant $(6.2 \%$ versus $2.9 \%, \mathrm{p}<0.001)$ and have fatal injury

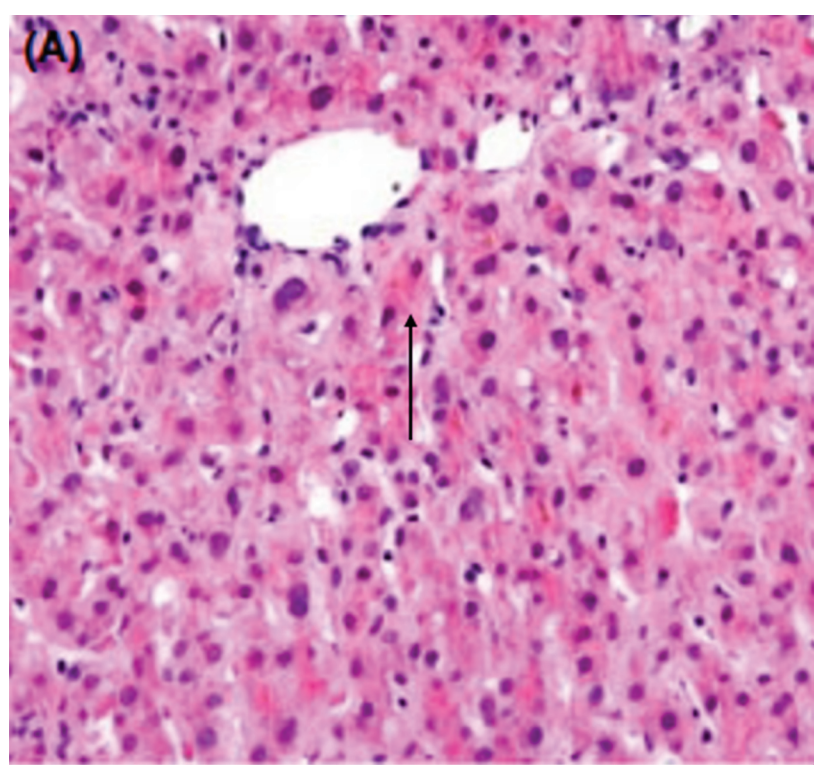

Fig 1. Haematoxylin and eosin stain showing bland cholestasis due to androgenic anabolic steroid use with bile staining of hepatocytes (arrow) and Kupffer cell cytoplasm. Original magnification 200x. Reprinted with permission from El Sherrif et al. ${ }^{35}$

(9\% versus $4 \%, \mathrm{p}<0.001){ }^{7}$ Furthermore, use of non-body building HDS was associated with a significantly higher need for liver transplant compared with conventional drugs (13\% versus $3 \%, \mathrm{p}<0.05) .{ }^{38}$ Worryingly, $7 \%$ of cases were attributed to HDS during the first 2 years of the American DILI registry compared with $20 \% 10$ years later. ${ }^{38}$ The American Acute Liver Failure Study Group (ALFSG) also recently reported that the prevalence of DILI-ALF/acute liver injury cases due to HDS increased between 1998-2007 and 2007-2015 (12.4 versus $21.1 \%, \mathrm{p}=0.047$ ). The HDS group had higher transplantation rates ( $56 \%$ versus $32 \%, \mathrm{p}<0.005)$ and lower transplant-free survival ( $17 \%$ versus $34 \%, \mathrm{p}=0.044)$ compared with those receiving conventional drugs. ${ }^{39}$

Specifically in hepatocellular DILI, a serum bilirubin of $\geq 3$ times ULN in the absence of biliary obstruction or Gilbert's syndrome is associated with a mortality of approximately $10 \%$ (range 5-50\%). ${ }^{40}$ This is also known as Hy's law, in recognition of the pioneering work done by Hyman Zimmerman - an American hepatologist. Hy's law has now been confirmed by European and American prospective DILI registries. ${ }^{7,8}$ Hy's law has also been adopted by the US FDA as a predictor of severe DILI during clinical trials, although in the interest of patient safety the FDA has lowered the hyperbilirubinaemia required to $\geq 2$ times ULN ('modified Hy's Law'). ${ }^{40}$ The US FDA states that 'finding one Hy's law case in the clinical trial database is worrisome; finding two is considered highly predictive that the drug has the potential to cause severe DILI when given to a larger population'. ${ }^{4}$

Predicting those who are likely to develop ALF in idiosyncratic DILI is difficult and even the application of Hy's law provides a specificity of only $44-67 \%$ and sensitivity of $83-90 \% .{ }^{8}$ In an attempt to improve this, the Spanish DILIN developed a composite score to predict ALF: AST $\geq 17.3$ times 
ULN; total bilirubin $\geq 6.6$ times ULN; and AST:ALT $\geq 1.5$ times ULN. These criteria are able to determine progression to ALF with a specificity of $82 \%$ and sensitivity of $80 \%{ }^{8}$ This composite score, however, needs to be validated in additional prospective studies.

\section{Diagnosis}

Since there are no diagnostic tests or biomarkers for idiosyncratic DILI, its diagnosis is made after stringently excluding other causes of liver disease, assessing the temporal association between use of drug and abnormal liver tests, looking for a characteristic clinical drug signature, and assessing improvement in liver tests upon drug withdrawal. Drug re-challenge is rarely indicated and cannot be advocated on ethical grounds. Nonetheless, one situation where a rechallenge may be justifiable is in the treatment of tuberculosis; however, this must only be done after carefully weighing the risks and benefits and in close liaison with a hepatologist. ${ }^{4}$ Finally, various causality assessment tools exist for DILI, of which the Roussel Uclaf Causality Assessment Method is the most used although it is far from perfect. ${ }^{41}$

Liver biopsy can be helpful in certain situations, particularly to exclude other causes of liver disease (eg alcohol, NAFLD). It also has a role if 'drug-induced' autoimmune hepatitis (AIH) is suspected and needs differentiating from idiosyncratic DILI. ${ }^{42}$ Portal and intra-acinar plasma cells, rosette formation and emperiopolesis are histological features that favour AIH $(\mathrm{p}<0.02)$, whereas portal neutrophils and intracellular (hepatocellular) cholestasis are more prevalent in DILI $(\mathrm{p}<0.02$; Table 2$) .{ }^{42}$ Nonetheless, this differentiation is based on retrospective data and, in the real world, differentiating 'drug-induced AIH' from idiosyncratic DILI may be far more difficult.

In case 2 (Box 1), a subsequent liver biopsy confirmed cirrhosis with lymphoplasmacytic portal inflammation and interface hepatitis. This was suggestive of AIH. While statins

\begin{tabular}{|c|c|c|c|}
\hline & $\begin{array}{l}\text { Idiosyncratic } \\
\text { DILI }\end{array}$ & $\begin{array}{l}\text { 'Drug } \\
\text { induced' AIH }\end{array}$ & $\begin{array}{l}\text { Idiopathic } \\
\text { AIH }\end{array}$ \\
\hline Histology & $\begin{array}{l}\text { Portal } \\
\text { neutrophils, } \\
\text { intracellular } \\
\text { cholestasis }\end{array}$ & $\begin{array}{l}\text { Interphase } \\
\text { hepatitis, } \\
\text { plasma cells }\end{array}$ & $\begin{array}{l}\text { Interphase } \\
\text { hepatitis, } \\
\text { plasma cells, } \\
\text { emperipolesis, } \\
\text { rosettes }\end{array}$ \\
\hline $\begin{array}{l}\text { Rash, } \\
\text { eosinophilia }\end{array}$ & $+1-$ & $+/-$ & - \\
\hline Fibrosis & $+1-$ & + & ++ \\
\hline $\begin{array}{l}\text { Response to } \\
\text { steroids }\end{array}$ & - & + & + \\
\hline $\begin{array}{l}\text { Relapse } \\
\text { on steroid } \\
\text { withdrawal }\end{array}$ & - & $+1-$ & + \\
\hline
\end{tabular}

have been associated with DILI, including 'drug-induced' $\mathrm{AIH},{ }^{43}$ it would have been very unusual for the statin to result in cirrhosis, especially within a year. ${ }^{43}$ This patient most likely had an underlying propensity to develop idiopathic $\mathrm{AIH}$ and the statin either unmasked or triggered this, or was an innocent bystander. She was treated with corticosteroids and azathioprine with good response.

\section{Management of DILI}

Prompt cessation of the culprit drug as soon as DILI is suspected is of paramount importance. There are also therapies for specific causes of DILI; for example, $\mathrm{N}$-acetylcysteine (NAC) for paracetamol overdose $\mathrm{e}^{44}$ and $\mathrm{L}$-carnitine for valproic acid overdose. $^{45}$

Corticosteroid therapy in DILI has a limited role and is reserved for those with immune-mediated DILI or 'druginduced' AIH. The former may only need a short course of steroids, whereas the latter, which is often indistinguishable from idiopathic $\mathrm{AIH}$, often requires prolonged courses of steroids and/or additional immunosuppression. ${ }^{46-49}$ As already stated, differentiating DILI from 'drug-induced' AIH can often be difficult and in such situations, after careful assessment of risks and benefits, a trial of corticosteroids may be warranted. Although ursodeoxycholic acid is often used in cholestatic DILI, its use remains non-evidence based. ${ }^{50}$

\section{Acute liver failure}

Case 3 (Box 1) presents a patient who has developed ALF. Pegylated interferon alpha use has not been associated with ALF. Hepatitis B and C flares were excluded by negative polymerase chain reaction; therefore, the most likely cause was clarithromycin-induced ALF. Clarithromycin has been associated with cholestatic DILI, particularly in older patients, and ALF has been reported. ${ }^{51}$ This patient was urgently listed for a liver transplant but unfortunately developed progressive cerebral oedema and died before he could receive a transplant.

Spontaneous survival with ALF secondary to idiosyncratic DILI (non-paracetamol) is poor with only $27.1 \%$ of patients alive at 3 weeks, ${ }^{14}$ in contrast to paracetamol-induced ALF where spontaneous survival is $64-66 \% .^{52}$ Therefore, it is crucial that, especially in idiosyncratic DILI, early discussion with a tertiary liver centre takes place at the first hint of ALF (worsening jaundice, coagulopathy (international normalised ratio $>1.5$ ) or development of hepatic encephalopathy (HE)). With transplantation the overall survival increases to $66.2 \%$, which demonstrates the necessity to rapidly identify those with ALF due to idiosyncratic DILI. ${ }^{8}$ The King's College criteria are most commonly used to prioritise patients with paracetamol and idiosyncratic DILI for liver transplant. ${ }^{53,54}$

Two earlier randomised controlled trials (RCTs) showed no benefit of corticosteroids in ALF and even demonstrated a poorer outcome in those with DILI. ${ }^{55,56}$ A more recent study from the ALFSG corroborated this, showing no improvement in overall survival in drug-induced, indeterminate or autoimmune $\operatorname{ALF}(61 \%$ versus $66 \%, \mathrm{p}=0.41) .^{57}$ In fact, lower survival was observed in those patients with the highest model for end stage liver disease scores $(>40)$ after corticosteroid therapy (30\% versus $57 \%, \mathrm{p}=0.03) .{ }^{57}$ 
NAC has a well-established role in paracetamol-induced $\mathrm{ALF}^{44}$ although it is now also recommended in selected cases of non-paracetamol ALF. A 2009 RCT in patients with non-paracetamol ALF demonstrated similar 3-week survival for patients given NAC versus placebo (70\% versus $66 \%$, $\mathrm{p}=0.283) .{ }^{58}$ However, transplant-free survival was significantly better for patients who received NAC compared with placebo ( $40 \%$ versus $27 \%, p=0.043$ ), but this was confined to those with early HE ( $52 \%$ versus $30 \%, p=0.010)$; whereas those with grade $3-4 \mathrm{HE}$ had no benefit with NAC (9\% versus $22 \%$, $\mathrm{p}=0.912) .{ }^{58}$ DILI was the most prevalent aetiology of ALF within this study.

\section{Conclusion}

DILI includes the whole spectrum from asymptomatic and transient elevation in liver tests to ALF. DILI due to paracetamol overdose and idiosyncratic drug reactions remain the most common causes of ALF in the western world. DILI is a diagnosis of exclusion and there is an urgent need to develop diagnostic biomarkers. Antimicrobials remain the most common cause of idiosyncratic DILI although recent studies show a significant increase in DILI due to herbal and dietary supplements. Hepatocellular DILI is more likely to progress to ALF compared with cholestatic or mixed DILI. The management of DILI is symptomatic with prompt cessation of the culprit drug and early referral for liver transplant upon the development of ALF, especially in those with idiosyncratic DILI.

\section{Conflicts of interest}

The authors declare no conflicts of interest related to this manuscript.

\section{References}

1 Kaplowitz N. Idiosyncratic drug hepatotoxicity. Nat Rev Drug Discov 2005;4:489-99.

2 Kaplowitz N. Drug-induced liver injury. Clin Infect Dis 2004;38(Suppl 2):S44-8.

3 Pham TV. Acetaminophen hepatotoxicity. In: Taylor M (ed). Gastrointestinal emergencies. Baltimore: Williams and Wilkins, 1997:371-88.

4 Verma S, Kaplowitz N. Diagnosis, management and prevention of drug-induced liver injury. Gut 2009;58:1555-64.

5 Sgro C, Clinard F, Ouazir K et al. Incidence of drug-induced hepatic injuries: a French population-based study. Hepatology 2002;36:451-5.

6 Björnsson ES, Bergmann OM, Björnsson HK, Kvaran RB, Olafsson S. Incidence, presentation, and outcomes in patients with drug-induced liver injury in the general population of Iceland. Gastroenterology 2013;144:1419-25.

7 Chalasani N, Bonkovsky HL, Fontana R et al. Features and outcomes of 899 patients with drug-induced liver injury: the DILIN prospective study. Gastroenterology 2015;148:1340-52.

8 Robles-Diaz M, Lucena MI, Kaplowitz N et al. Use of Hy's law and a new composite algorithm to predict acute liver failure in patients with drug-induced liver injury. Gastroenterology 2014;147:109-18.

9 de Abajo FJ, Montero D, Madurga M, García Rodríguez LA. Acute and clinically relevant drug-induced liver injury: a population based case-control study. Br J Clin Pharmacol 2004;58:71-80.

10 Andrade RJ, Lucena MI, Fernández MC et al. Drug-induced liver injury: an analysis of 461 incidences submitted to the Spanish registry over a 10-year period. Gastroenterology 2005;129:512-21.

11 Suk KT, Kim DJ, Kim CH et al. A prospective nationwide study of drug-induced liver injury in Korea. Am J Gastroenterol 2002;107:1380-7.
12 Bernal W, Wendon J. Acute liver failure. N Engl J Med 2013;369:2525-34

13 Lee WM. Acute liver failure in the United States. Semin Liver Dis 2003;23:217-26.

14 Reuben A, Koch DG, Lee WM, Acute Liver Failure Study Group. Drug-induced acute liver failure: results of a US multicenter, prospective study. Hepatology 2010;52:2065-76.

15 Bénichou C. Criteria of drug-induced liver disorders. Report of an international consensus meeting. J Hepatol 1990;11:272-6.

16 Navarro V. Hepatic adverse event nomenclature document. Available online at www.fda.gov/downloads/Drugs/ScienceResearch/.../ ucm080365.ppt [Accessed 7 September 2016].

17 Salvo F, Polimeni G, Moretti U et al. Adverse drug reactions related to amoxicillin alone and in association with clavulanic acid: data from spontaneous reporting in Italy. J Antimicrob Chemother 2007;60:121-6.

18 Beraldo DO, Melo JF, Bonfim AV et al. Acute cholestatic hepatitis caused by amoxicillin/clavulanate. World J Gastroenterol 2013;19:8789-92.

19 Obermayer-Straub P, Manns MP. Immunological mechanisms in liver injury. In: Kaplowitz N and DeLeve L (eds). Drug-induced liver disease. Boca Raton, FL: CRC Press, 2003:125-149.

20 Abboud G, Kaplowitz N. Drug-induced liver injury. Drug Saf 2007;30:277-94.

21 Sakaan SA, Twilla JD, Usery JB et al. Nitrofurantoin-induced hepatotoxicity: a rare yet serious complication. South Med J 2014;107:107-13.

22 Amacher DE, Chalasani N. Drug-induced hepatic steatosis. Semin Liver Dis 2014;42:205-14.

23 Gorayski P, Thompson CH, Subhash HS, Thomas AC. Hepatocellular carcinoma associated with recreational anabolic steroid use. Br J Sports Med 2008;42:74-5.

24 Musumba CO. Review article: the associated between nodular regenerative hyperplasia, inflammatory bowel disease and thiopurine therapy. Aliment Pharmacol Ther 2013;38:1025-37.

25 Amacher DE. Female gender as a susceptibility factor for drug-induced liver injury. Hum Exp Toxicol 2014;33:928-39.

26 Lucena MI, Andrade RJ, Kaplowitz N et al. Phenotypic characterization of idiosyncratic drug-induced liver injury: the influence of age and sex. Hepatology 2009;49:2001-9.

27 Lammert C, Einarsson S, Saha C et al. Relationship between daily dose of oral medications and idiosyncratic drug-induced liver injury: search for signals. Hepatology 2008;47:2003-9.

28 Russo MW, Galanko JA, Shrestha R, Fried MW, Watkins P. Liver transplantation for acute liver failure from drug induced liver injury in the United States. Liver Transpl 2004;10:1018-23.

29 Björnsson E, Davidsdottir L. The long-term follow-up after idiosyncratic drug-induced liver injury with jaundice. J Hepatol 2009;50:511-7.

30 Rosenberg P, Urwitz H, Johannesson A et al. Psoriasis patietns with diabetes type 2 are at high risk of developing liver fibrosis during methotrexate treatment. J Hepatol 2007;46:1111-8.

31 Chien JY, Huang RM, Wang JY et al. Hepatitis C virus infection increases hepatitis risk during anti-tuberculosis treatment. Int $J$ Tuberc Lung Dis 2010;14:616-21.

32 Wong WM, Wu PC, Yuen MF et al. Antituberculosis drug-related liver dysfunction in chronic hepatitis B infection. Hepatology 2000;31:201-6.

33 Verma S, Kaplowitz N. Hepatotoxicity of antituberculosis drugs. In: Kaplowitz N and DeLeve LD (eds). Drug-induced liver disease, 3rd edn. London: Academic Press, 2013:483-504.

34 Daly AK, Donaldson PT, Bhatnagar P et al. HLA-B ${ }^{\star} 5701$ genotype is a major determinant of drug-induced liver injury due to flucloxacillin. Nat Genet 2009;41:816-9.

35 El Sherrif Y, Potts JR, Howard MR et al. Hepatotoxicity from anabolic androgenic steroids marketed as dietary supplements: contribution from ATP8B1/ABCB11 mutations? Liver Int 2013;33:1266-70. 
36 Chen M, Borlak J, Tong W. High lipophilicity and high daily dose of oral medications are associated with significant risk for druginduced liver injury. Hepatology 2013;58:388-96.

37 Fontana RJ. Pathogenesis of idiosyncratic drug-induced liver injury and clinical perspectives. Gastroenterology 2014;146:914-28.

38 Navarro VJ, Barnhart H, Bonkovsky HL et al. Liver injury from herbals and dietary supplements in the US Drug-Induced Liver Injury Network. Hepatology 2014;60:1399-408.

39 Hillman L, Gottfried M, Whitsett $\mathrm{M}$ et al. Clinical features and outcomes of complementary and alternative medicine induced acute liver failure and injury. Am J Gastroenterol 2016;111:958-95.

40 United States Food and Drug Administration. Drug-induced liver injury: premarketing clinical evaluation. Silver Spring, MD: US FDA, 2009. Available online at www.fda.gov/downloads/ Drugs/GuidanceComplianceRegulatoryInformation/Guidances/ UCM174090.pdf [Accessed 7 September 2016].

41 Danan G, Benichou C. Causality assessment of adverse reactions to drugs. A novel method based on the conclusions of international consensus meetings: application to drug-induced liver injuries. J Clin Epidemiol 1993;46:1323-30.

42 Suzuki A, Brunt EM, Kleiner DE et al. The use of liver biopsy evaluation in discrimination of idiopathic autoimmune hepatitis versus drug-induced liver injury. Hepatology 2011;54:931-9.

43 Russo MW, Hoofnagle JH, Gu J et al. Spectrum of statin hepatotoxicity: experience of the drug-induced liver injury network. Hepatology 2014;60:679-86.

44 Polson J, Lee WM, American Association for the Study of Liver Disease. AASLD position paper: the management of acute liver failure. Hepatology 2005;41:1179-97.

45 Bohan TP, Helton E, McDonald I et al. Effect of L-carnitine treatment for valproate-induced hepatotoxicity. Neurology 2001;56:1405-9.

46 Björnsson ES, Talwalkar J, Treeprasertsuk S et al. Drug-induced autoimmune hepatitis: clinical characteristics and prognosis. Hepatology 2010;51:2040-8.

47 Weiler-Normann C, Schramm C. Drug induced liver injury and its relationship to autoimmune hepatitis. J Hepatol 2011;55:747-9.

48 Yeong T, Lim KHJ, Goubet S, Parnell N, Verma S. Natural history and outcomes in drug induced autoimmune hepatitis. Hepatol Res 2016;46:E79-88.
49 A Heurgué, Bernard-Chabert B, Diebold M et al. Drug-induced autoimmune hepatitis: a frequent disorder. Gut 2007;56(Suppl 3):A271.

50 Studniarz M, Czubkowski P, Cielecka-Kuszyk J et al. Amoxicillin/ clavulanic acid-induced cholestatic liver injury after pediatric liver transplantation. Ann Transplant 2012;17:128-31.

51 Maggi P, Solarino B, Cassano P et al. Fatal fulminant hepatitis following administration of clarithromycin in a patient chronically treated with antipsychotic drugs. Immunopharmacol Immunotoxicol 2013;35:191-4.

52 Fontana RJ. Acute liver failure including acetaminophen overdose. Med Clin North Am 2008;92:761-94.

53 O'Grady JG, Alexander GJ, Hayllar KM, Williams R. Early indicators of prognosis in fulminant hepatic failure. Gastroenterology 1989;97:439-45.

54 McPhail MJ, Wendon JA, Bernal W. Meta-analysis of performance of King's College Hospital Criteria in prediction of outcome in non-paracetamol-induced acute liver failure. J Hepatol 2010;53:492-9.

55 Raklea J, Mosley JW, Edwards VM, Govindarajan S, Alpert E. A double-blinded, randomized trial of hydrocortisone in acute hepatic failure. The Acute Hepatic Failure Study Group. Dig Dis Sci 1991;36:1223-8.

56 Randomised trial of steroid therapy in acute liver failure. Report from the European Association for the Study of the Liver (EASL). Gut 1979;20:620-3.

57 Karkhanis J, Verna EC, Chang MS et al. Steroid use in acute liver failure. Hepatology 2014;59:612-21.

58 Lee WM, Hynan LS, Rossaro L et al. Intravenous N-acetylcysteine improves transplant-free survival in early stage non-acetaminophen acute liver failure. Gastroenterology 2009;137:856-64.

Address for correspondence: Dr S Verma, Medical School Teaching Building, Room 2.17, Brighton and Sussex Medical School, Falmer, Brighton BN1 9PX, UK.

Email: s.verma@bsms.ac.uk 